Gut, 1975, 16, 132-136

\title{
Function and structure of the mucosa of continent ileostomy reservoirs in dogs
}

\author{
B. PHILIPSON ${ }^{1}$, N. G. KOCK, J. W. L. ROBINSON, H. MENGE ${ }^{2}$, AND \\ V. MIRKOVITCH
}

From Surgical Department III, Sahlgren's Hospital, Göteborg, Chirurgische Klinik A, Kantonsspital, Zürich, and the Department of Experimental Surgery, Hopital Cantonal Universitaire, Lausanne

SUMMARY Continent ileostomy reservoirs were constructed in dogs. Nine to 29 days after formation of the pouches, they were excised together with a loop of adjacent ileum for biochemical and morphological assessment. The villous and epithelial cell heights were reduced, whereas villous width and mitotic index were increased. The mucosa of the pouch was able to absorb phenylalanine and $\beta$-methyl-glucoside against a concentration gradient, although this absorptive capacity was lower than that of normal ileum. Similar considerations applied to oxygen consumption. The results indicate that the mucosa of the pouch undergoes transformation, but the integrity of the epithelial layer is maintained.

Recently a 'continent ileostomy' was devised (Kock, 1969, 1973; Kock, Darle, Kewenter, Myrvold, and Philipson, 1974) and introduced as an alternative to the conventional ileostomy in man. The continent ileostomy utilizes an intraabdominal reservoir constructed from the distal ileum for collection and temporary storage of the intestinal contents.

Clinical follow-up studies in patients with continent ileostomies have revealed morphological changes in the mucosa of the intraabdominal reservoir, which included decreased villous height and increased epithelial turnover (Philipson, Brandberg, Jagenburg, Kock, Lager, and Åhrén, 1975). However, the ability of the intestines of these patients to absorb L-phenylalanine, D-xylose, fat, and vitamin $B_{12}$ is the same as that of patients with conventional ileostomies (Jagenburg, Dotevall, Kewenter, Kock, and Philipson, 1971; Jagenburg, Kock, and Philipson, 1975). In spite of the presence of an increased number of microorganisms (Brandberg, Kock, and Philipson, 1972; Philipson et al, 1975), local vitamin $B_{12}$ absorption has been demonstrated in the reservoirs of some patients (Jagenburg et al, 1975).

\footnotetext{
${ }^{1}$ Reprint requests to B. Philipson, Surgical Department III, Sahlgrenska sjukhuset, S 41345 Göteborg, Sweden ${ }^{2}$ On leave of absence from the Universitätsklinik, Marburg/Lahn Received for publication 4 December 1974.
}

The absorptive capacity of the mucosa used for the construction of the reservoir is difficult to study in vivo under standardized conditions. For this reason, animal experiments have been performed to estimate in vitro the transport of amino acids and sugars by the mucosa of the reservoir.

\section{Materials and Methods}

SURGICAL PROCEDURE

Nineteen mongrel dogs of both sexes weighing 9.5-17.5 kg were used. Paramedian laparotomy was performed under pentobarbital anaesthesia and sterile conditions. The ileum was divided about $7 \mathrm{~cm}$ from the ileocaecal valve, and the distal stump of ileum was closed. The construction of the ileostomy reservoir corresponded exactly to that described previously for clinical use (Kock, 1973). Briefly, a loop of ileum was formed into a $U$, the walls of the $U$ were sewn together, and the loop was opened longitudinally. The terminal unsplit segment was employed for the construction of the nipple valve. Then the bottom of the $U$ was folded up and the free edges were sewn together to form a hermetic pouch. The completed pouch was then placed in the abdomen and fixed in position. The outlet was passed through the abdominal wall by means of a separate incision and an ileostomy was created. Before closure of the abdomen, a catheter was 
inserted into the pouch for volume registration and subsequent drainage.

After operation, the dogs were fed by parenteral nutrition until the fifth day, but started to drink gradually two days after the operation. On the third or fourth day, the permanent catheter was removed; thereafter the reservoir was emptied three times a day by insertion of a catheter through the outlet. All dogs were continent for both gas and faeces.

After an observation time of nine to 29 days, the dogs were re-anaesthetized. After inspection of the reservoir and the proximal intestine, the reservoir was intubated and emptied, and its volume was determined by insufflation of saline through the outlet. The reservoir was then removed together with about $50 \mathrm{~cm}$ of the small intestine proximal to it, and the dogs were sacrificed. Samples of the whole intestinal wall were taken from the reservoir and the excised intestine for biochemical and morphological investigation.

\section{MORPHOLOGICAL EVALUATION}

Tissue samples were fixed in formalin, cut $(6 \mu)$, and stained with haematoxylin/eosin. Morphometric evaluation was performed by measuring the villous height, villous width, crypt length, and also the epithelial cell height at the middle of a villus. For these studies, only samples cut parallel to the villus/crypt axis were employed. In addition, the numbers of mitoses per crypt were counted and expressed as a percentage of the total number of cells in the crypt (the mitotic index).

BIOCHEMICAL EXAMINATIONS

The absorptive capacity of the intestinal mucosa was determined in vitro using incubation techniques that have previously been described in detail (Robinson and Mirkovitch, 1972). Briefly, the samples of mucosa of the reservoir and the proximal intestine were separated from their underlying muscular layers and cut into slices for incubation. They were incubated for one hour at $37^{\circ} \mathrm{C}$ in ${ }^{14} \mathrm{C}$-labelled $\mathrm{L}$-phenylalanine or $\beta$-methyl-D-glucoside ( $1 \mathrm{mM}$ solutions in Krebs bicarbonate buffer), and the uptake at the end of the incubation was calculated from the radioactivity absorbed. The extracellular space was determined by incubation of parallel samples in ${ }^{14} \mathrm{COOH}$-inulin and the water content of the tissues was calculated after desiccation of strips of mucosa overnight at $110^{\circ} \mathrm{C}$. From these data, the distribution ratio between intra- and extracellular water was computed.

Parallel samples of mucosa were employed for the determination of oxygen consumption and acid phosphatase release. The slices were incubated in a Krebs phosphate buffer containing $2 \mathrm{mg} / \mathrm{ml}$ glucose in Warburg flasks connected to a Gilson differential respirometer. The flasks were equilibrated for 30 minutes, and the oxygen consumption was determined over the next 50 minutes, readings being taken every 10 minutes, and the regression line between the measurements was computed. The results were corrected for standard temperature and pressure. At the end of the incubation, the total acid phosphatase liberated from the tissue into the medium was determined using the method of Fishman and Lerner (1953). The incubated tissues were dried overnight, and both measurements, oxygen consumption and acid phosphatase release, were expressed in terms of dry tissue.

\section{STATISTICS}

For geographical reasons (dogs being operated in Zürich or Göteborg and samples being removed for study in Lausanne), it was impracticable to excise and study a control sample of the intestine of the same dog at the moment of construction of the pouch. Consequently, the functional results were compared with the populations of normal values accumulated in our laboratory from other experimental series, whilst the morphometric measurements were compared with those of terminal ileum excised from dogs of similar weight and age. All results were expressed as means \pm SEM and comparisons between the experimental groups were performed by the $t$ test.

\section{Results}

Of the 19 dogs in which the pouches were constructed, eight survived long enough for the characteristic changes in the reservoir to have developed. In stark contrast to the success of the operation in man, a high mortality rate was encountered in our experiments with dogs. This must be attributed to the difficulties in immediate postoperative care of animals, which subsequently led to deterioration in their condition. In addition a number of dogs had to be sacrificed due to extensive wound infection.

The volume of the reservoir at the time of construction was $20-40 \mathrm{ml}$. At sacrifice, this volume had increased to 150 to $400 \mathrm{ml}$ (mean $280 \mathrm{ml}$ ). The aspect of the pouch at this time is shown in figure 1. The intestine immediately above the pouch was generally dilated and full of fluid. Thus it is conceivable that the intestine initially responds to the construction of the pouch in a similar manner as to partial occlusion, at least until the volume of the pouch is sufficient to drain the whole intestine.

Under the dissection microscope, it was observed that the mucosa of the reservoir possessed mainly finger-like villi that were shorter than normal, 


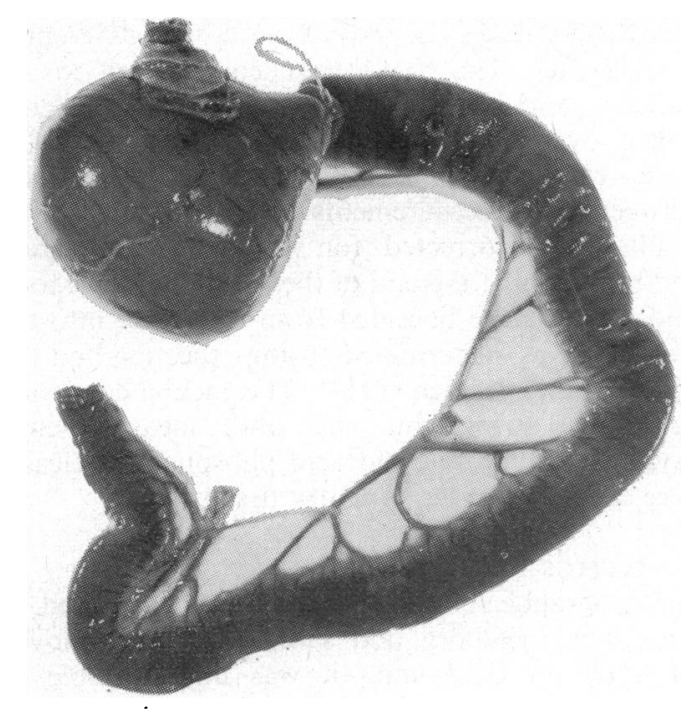

Fig 1 Macroscopic aspect of dog continent ileostomy with adjacent intestine at time of excision.

together with occasional mosaic forms. This was also true of the intestine proximal to the pouch, though to a lesser degree. This differs slightly from the picture of human continent ileostomy reservoirs, where a mixture of finger- and leaf-shaped villi was encountered (Philipson et al, 1975).

These observations were confirmed by the morphometric evaluations (fig 2) where a generalized reduction in the villous height is revealed. Paired data analysis showed that this reduction was more pronounced in the mucosa of the reservoir than in the intestine above it. In addition, there was an increase in villous width and a reduction in epithelial cell height. The crypt length, both in the pouch and above it, was very variable, some dogs displaying very elongated crypts. The mitotic index of the reservoir mucosa was $3.05 \pm 0 \cdot 194$, whereas that of the intestine proximal to it was $2.25 \pm 0.193$. These two values obtained from eight dogs are significantly different $(t=2.93 ; P<0.02)$.

There was finite but significantly reduced phenylalanine and $\beta$-methyl-glucoside transport in the mucosa of the pouch and of the intestine proximal to it (fig 3). However, there were no significant differences between the mucosa of the pouch and the intestine above it. The same was true for oxygen consumption and acid phosphatase release, which were both lower than the control values, though again there were no differences between the two regions of the mucosa.

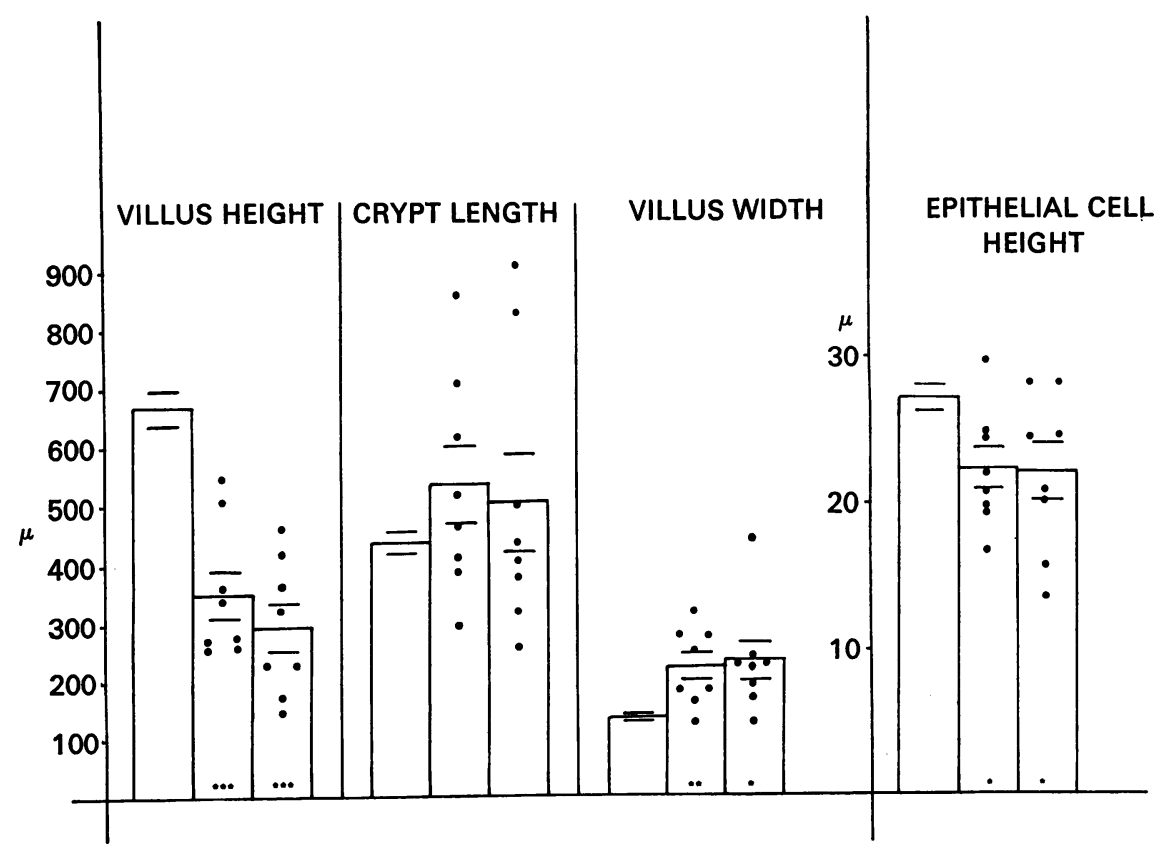




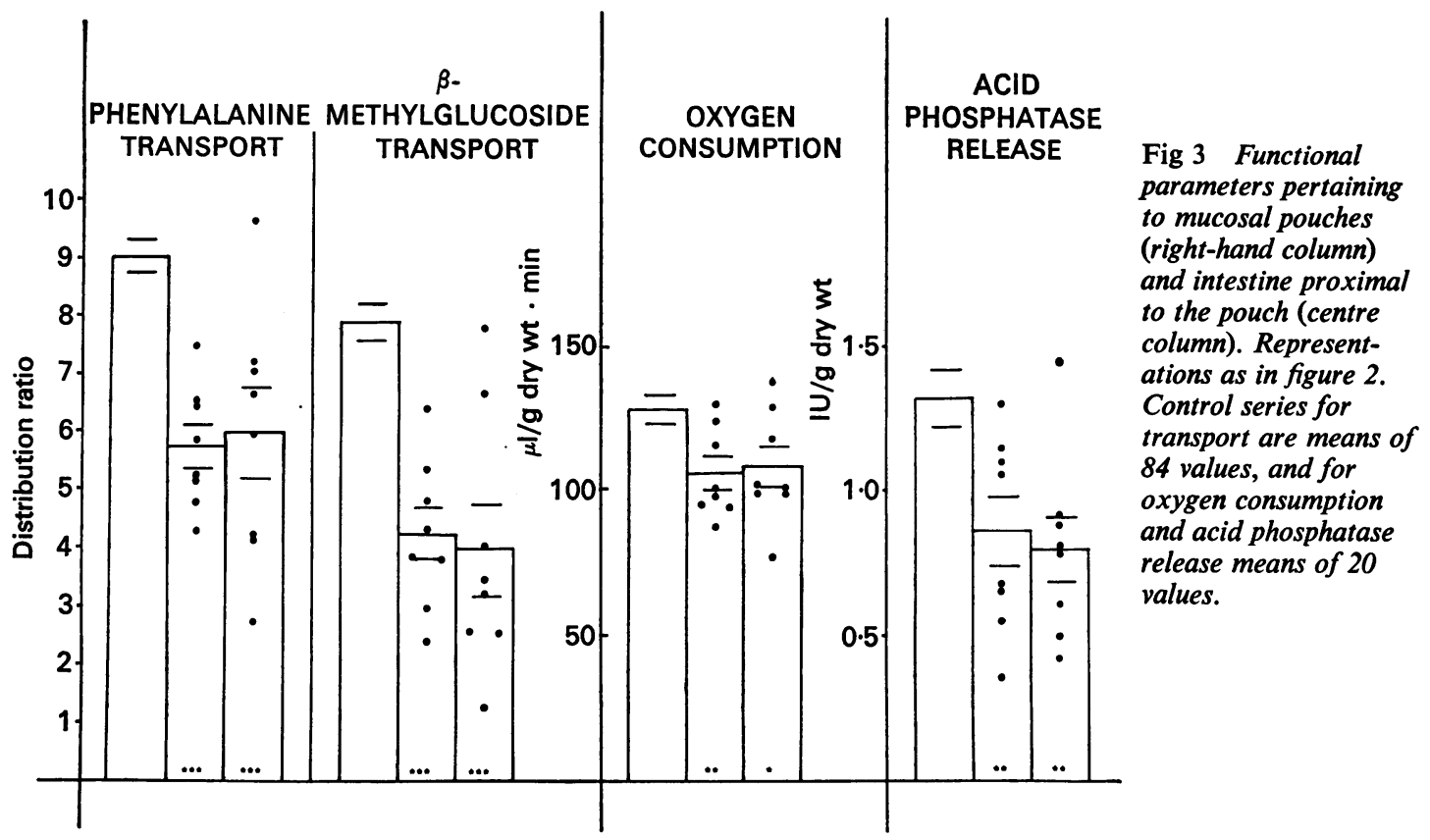

\section{Discussion}

Even though much information has been obtained from biopsy samples of continent ileostomy pouches in man, a better understanding of the function of the mucosa of these pouches has required the development of an animal model. It would be feasible in patients to ascertain the absorptive properties of the intestine above the pouch by appropriate perfusion techniques in vivo, but a precise evaluation of the absorption by the mucosa of the reservoir itself would meet with methodological problems, primarily because of difficulties in mixing the fluid efficiently within the pouch. Thus in the present study, we have chosen to examine in dogs the absorptive characteristics of the mucosa of the pouch and its adjacent ileum in vitro.

The validity of this approach is apparent by the marked similarities between the morphological responses revealed in dogs and those found in biopsy samples from patients (Philipson et al, 1975). The height of the villi of the pouch mucosa was markedly reduced; the villous width was increased; and the mitotic index was considerably enhanced.

Changes in villous height may be explained by alterations in turnover in response to harmful intraluminal factors. The greater crypt length 3 encountered in some samples, together with the increased mitotic index, is suggestive of a higher turnover. A similar hyperregenerative transformation with shorter villi has been reported by Riecken, Bloch, Menge, Idelberger, Kramer, Miller, and Lorenz-Meyer (1972), who instilled lactic acid as an intraluminal toxic agent into the jejunum of the rat. A further indication of this type of injury is provided by the increased villous width and the flattening of the epithelium. It is not yet certain which factor is responsible for initiating this transformation: the exposure of the mucosa to unusual bacterial flora (Brandberg et al, 1972) or the continual presence of a fluid probably containing metabolites and bacterial toxins.

The present investigations demonstrate that the mucosa of the reservoir is capable of transporting sugars and amino acids against considerable concentration gradients, though their absorptive capacity is nevertheless lower than that of the normal dog ileum. A similar reduction was observed in the intestine proximal to the pouch (where the changes are probably due to the rapid dilatation of the reservoir in the dog, caused by insufficient drainage of the pouch). These findings are readily explained in terms of the reduced villous height, and therefore of the smaller number of active absorbing cells in a given sample of mucosa, since it has been demonstrat- 
ed in a previous study that there is a close correlation between villous height and absorptive capacity (Robinson, Haroud, Luisier, Winistörfer, and Mirkovitch, 1974). The same explanation applies to the reduction in oxygen consumption. The release of acid phosphatase provides an index of the cellular integrity, cellular damage being reflectep by an increase in this parameter (Abbott and Sell, 1968). The reduction in the liberation observed here, which parallels the decrease in the other biochemical evaluations, is a further indication of the presence of an intact, though numerically reduced epithelial population.

In conclusion, the present study suggests that the mucosa of the reservoir undergoes a hyperregenerative transformation, probably in response to the exogenous stress caused by its contents. The biochemical studies indicate nevertheless that although the number of active absorbing cells is reduced, their functional integrity is maintained.

This study was supported by the Swedish Medical Research Council (Project Numjer B75/17X/ 577/11A)

We are most grateful to the Swiss Consulate in Göteborg for their advice and collaboration, without which this project could never have been completed. The stay of one of us (N.G.K.) in Zürich was supported in part by the Roche Research Foundation for scientific exchange and biomedical collaboration in Switzerland. It is a pleasure to acknowledge the skilful technical assistance of Harriet Andersson, Elizabet Lindholm, and Ingela Rosén in Göteborg and of Monique Augstburger, Hélène Capt, Dominique Mettraux, Lilian
Schnell, Georges Stauffer, and Janine Vouron in Lausanne. We are grateful also to Agneta Särén in Göteborg and Winefred Happee in Lausanne for secretarial aid.

\section{References}

Abbott, W. M., and Sell, K. W. (1968). Functional in-vitro analysis as a key to organ storage. In Organ Perfusion and Preservation, edited by J. C. Norman, pp. 487-503. Appleton-CenturyCrofts, New York.

Brandberg, §̊., Kock, N. G., and Philipson, B. (1972). Bacterial flora in intra-abdominal ileostomy reservoir. Gastroenterology, 63, 413-416.

Fishman, W. H., and Lerner, F. (1953). A method for estimating serum acid phosphatase of prostatic origin. J. biol. Chem., 200, 89-97.

Jagenburg, R., Dotevall, G., Kewenter, J., Kock, N. G., and Philipson, B. (1971). Absorption studies in patients with 'intraabdominal ileostomy reservoirs' and in patients with conventional ileostomies. Gut, 12, 437-441.

Jagenburg, R., Kock, N. G., and Philipson, B. (1975). Vitamin $B_{12}$ absorption in patients with continent ileostomy. Scand. J. Gastroent in press.

Kock, N. G. (1969). Intra-abdominal 'reservoir' in patients with permanent ileostomy. Arch. Surg., 99, 223-231.

Kock, N. G. (1973). Continent ileostomy. Progr. Surg., 12, 180-201.

Kock, N. G., Darle, N., Kewenter, J., Myrvold, H., and Philipson, B. (1974). The quality of life after proctocolectomy and ileostomy: a study of patients with conventional ileostomies converted to continent ileostomies. Dis. Colon. Rect., 17, 287-292.

Philipson, B., Brandberg, A.., Jagenburg, R., Kock, N. G., Lager, I., and Åhrén, C. (1975). Studies of mucosal morphology, bacteriology and absorption in intraabdominal ileostomy reservoir. Scand. J. Gastroent., in press.

Riecken, E. O., Bloch, R., Menge, H., Idelberger, K., Kramer, F., Miller, B., and Lorenz-Meyer, H. (1972). Morphologische und funktionelle Befunde nach Milchsäuredauerinfusion in das Jejunum der Ratte. Z. Zellforsch., 132, 107-129.

Robinson, J. W. L., Haroud, M., Luisier, A. L., Winistörfer, B., and Mirkovitch, V. (1974). Fonction et structure de la muqeuse intestinale aprés résection étendue de l'intestin grêle chez le chien. Biomedicine Express, 21, 71-76.

Robinson, J. W. L., and Mirkovitch, V. (1972). The recovery of function and microcirculation in small intestinal loops following ischaemia. Gut, 13, 784-789. 